\title{
Corela
}

Cognition, représentation, langage

HS-16 | 2015

Diversité des pratiques de recherche en science du langage

\section{Analyse typologique et syntaxique du génitif wolof}

\section{Maximilien Guérin}

\section{(2) OpenEdition}

Journals

Édition électronique

URL : http://journals.openedition.org/corela/3853

DOI : $10.4000 /$ corela.3853

ISSN : 1638-573X

Éditeur

Cercle linguistique du Centre et de l'Ouest - CerLICO

Référence électronique

Maximilien Guérin, «Analyse typologique et syntaxique du génitif wolof », Corela [En ligne],

HS-16 | 2015, mis en ligne le 29 juin 2015, consulté le 07 mai 2019. URL : http://

journals.openedition.org/corela/3853; DOI : 10.4000/corela.3853

Ce document a été généré automatiquement le 7 mai 2019.

\section{(c) (i) (3) (2)}

Corela - cognition, représentation, langage est mis à disposition selon les termes de la licence Creative Commons Attribution - Pas d'Utilisation Commerciale - Partage dans les Mêmes Conditions 4.0 International. 


\title{
Analyse typologique et syntaxique du génitif wolof
}

\author{
Maximilien Guérin
}

\section{Approche typologique du génitif}

1 Le génitif en wolof ${ }^{1}$ est une construction qui a reçu des analyses très diverses, dont beaucoup restent parcellaires ou basées sur des exemples pouvant paraître douteux. Avant d'entamer une description du génitif wolof, il convient donc de se pencher sur l'ensemble des analyses proposées afin d'en mesurer la pertinence et d'en déterminer les limites. Il convient également de donner une perspective typologique à la description afin de comprendre où se situe le génitif du wolof parmi les différentes constructions possibles attestées dans les langues.

\subsection{Définitions}

2 La première question que l'on est tenu de se poser est : qu'est-ce que le génitif ? Dans cet article nous entendrons par " génitif », ou " construction génitive », la construction dans laquelle un nom est déterminé par un syntagme nominal. Le nom déterminé sera appelé «nom tête » et le syntagme déterminant sera appelé «syntagme génitif» (Dryer, 2007 : 178). Une telle définition, essentiellement morphosyntaxique, implique deux choses :

- La construction génitive ne se limite pas aux relations de possession, mais englobe généralement toutes sortes de relations entre un nom et un syntagme nominal : relation familiale (le frère de Jean), possession (le livre de Jean), partie d'une entité (le bras de Jean), relations abstraites (l'anniversaire de Jean).

- La construction génitive est distincte du cas génitif présent dans les langues à cas. En effet, bien que le cas génitif soit une manière possible de relier un nom tête à un syntagme génitif, il a généralement des emplois qui ne correspondent pas à la définition que nous avons donnée de la construction génitive. En finnois, par exemple, le cas génitif est un cas ne 
faisant que refléter certaines relations de dépendances syntaxiques entre les constituants (Mahieu, 2013). Dans le syntagme äärettömän yksinkertainen ('infiniment simple'), le mot äärettömä ('infini') est au cas génitif alors qu'aucun nom n'est présent dans le syntagme. Réciproquement, toute construction génitive n'est pas marquée morphologiquement au génitif.

Bien que la construction génitive ne se limite pas aux relations de possession, il est possible de trouver une certaine unité dans ce type de construction. En effet, elle «renvoie fondamentalement à la notion de participation d'une entité (conventionnellement désignée comme le possédé) à la sphère personnelle ${ }^{2} \mathrm{~d}^{\prime} u n$ individu (conventionnellement désigné comme le possesseur)»(Creissels, 2001). Cette sphère personnelle se construit autour de trois prototypes, qui sont la relation d'un individu: aux parties de son corps ; à des parents ; à des objets courants. Par extension, elle sert à marquer des relations avec des noms abstraits: agents (la parole de Paul), patients (la destruction de la ville), etc.

\subsection{Typologie des constructions génitives et possessives}

Il existe un nombre limité de constructions possibles pour le génitif à travers les langues. En se basant sur Dryer (2007: 177-182), nous pouvons élaborer une typologie des constructions génitives reposant sur la nature, l'ordre et l'organisation des éléments au sein de la construction :

- Le syntagme génitif et le nom tête sont juxtaposés

- Le syntagme génitif précède le nom tête

- Le nom tête précède le syntagme génitif

- Le syntagme génitif porte une marque du génitif

- La marque du génitif est un affixe génitif

- La marque du génitif est une adposition génitive

- La marque du génitif est un clitique génitif

- Le nom tête porte une marque du génitif

- La marque du génitif est un affixe génitif qui s'accorde avec le syntagme génitif

- La marque du génitif est un affixe génitif invariable

- La valeur du génitif est portée par un mot génitif

- Le mot génitif sépare le syntagme génitif et le nom tête

- Le mot génitif est une adposition ou est indéterminé

- Le mot génitif est un pronom qui s'accorde avec le syntagme génitif

- Le mot génitif ne sépare pas le syntagme génitif et le nom tête

- Le mot génitif est une adposition adjacente au syntagme génitif

- Le mot génitif est un pronom adjacent au nom tête

- Le mot génitif est un pronom adjacent au syntagme génitif

- Différentes constructions sont utilisées simultanément

Nous parlons de construction possessive lorsque le possesseur (le syntagme génitif dans la construction génitive) est une forme pronominale. Tout comme la construction génitive, il existe un nombre limité de constructions possibles pour le possessif à travers les langues. En se basant sur Dryer (2007: 182-183) et Creissels (2006: 141-160), nous 
pouvons élaborer une typologie des constructions génitives reposant sur les mêmes facteurs que ceux énoncés précédemment.

- La construction possessive est identique à la construction génitive

- La construction possessive est différente de la construction génitive

- La possession est marquée par un " pronom » possessif

- La possession est marquée par un affixe possessif

6 La construction possessive serait donc une pronominalisation de la construction génitive. Cependant il faut noter qu'il existe certaines situations où il est impossible de faire alterner une construction génitive avec une construction possessive : la chambre de Jean / sa chambre ; la chambre d'hôtel /*sa chambre.

\subsection{Génitif et définitude}

7 On pourrait penser, dans un premier temps, que construction génitive/possessive et déterminant défini sont redondants puisque la construction génitive/possessive spécifie le sens du nom tête. En effet, dans certaines langues, on observe une incompatibilité entre déterminant défini et construction génitive/possessive, comme en espagnol (1a-b) ( cf. Haspelmath, 1999 : 227).

8 (1a) mi libro mon livre 'mon livre'

$9 \quad(1 \mathrm{~b}) *$ el mi libro le mon livre

10 Cependant, dans plusieurs langues, on observe la possibilité de cumuler les deux dans un syntagme. C'est notamment le cas du grec moderne ou du basque (2a-b) ${ }^{3}$ (ibid. : 228).

11 (2a) amaren diru- $a$ mère.GEN argent-le 'l'argent de la mère'

12 (2b) zuen liburu-ak tu.GEN livre-les 'tes livres'

13 Il semblerait que l'incompatibilité entre déterminant défini et construction génitive/ possessive ne soit qu'une tendance due à des motivations d'économie ${ }^{4}$. «Possessed NPs have a very high chance of being definite for semantic and pragmatic reasons, and the resulting redundancy is exploited by some languages in which possessed NPs lack a definite article» (ibid. : 239). De plus, il est possible de faire ressortir les trois universaux suivants (ibid. : 234-235) : si le SN possédé contient un article défini, alors les autres SN également; si le SN possédé dont le possesseur est préposé contient un article défini, alors le SN possédé dont le possesseur est postposé en contient un également; si le SN possédé dont le nom tête est un terme de parenté contient un article défini, alors le SN possédé dont le nom tête n'est pas un terme de parenté en contient un également.

14 La forme construite du nom représente un cas particulier d'incompatibilité de déterminant défini et construction génitive/possessive. Ce type de construction, attesté 
dans les langues sémitiques, est caractérisé par plusieurs propriétés, dont les principales sont (Kihm, $2000: 152)$ :

- Le nom tête précède le syntagme génitif;

- Le nom tête ne peut pas être directement modifié par un déterminant, un adjectif ou une proposition relative; si l'un de ces deux derniers modifieurs est présent, il se trouve sur le bord droit de la construction ;

- La valeur de définitude du syntagme génitif (ouvertement marquée par un déterminant s'il est défini, non marquée dans les autres cas) s'étend sur l'ensemble de la construction;

- Lorsque plusieurs syntagmes génitifs se succèdent, chacun d'entre eux, à l'exception du dernier, est une tête pour le syntagme génitif qui suit;

- La relation entre le nom tête et le syntagme génitif n'est pas marquée par un élément tel qu'une adposition ou une marque casuelle.

Par exemple en hébreu moderne :

(3) delet beyt morat ha-kita ha-yafa

porte maison enseignant la-classe la-belle

'la belle porte de la maison de l'enseignant de la classe'

Par ailleurs, il faut signaler que la forme construite du nom est également utilisée pour les constructions possessives (Creissels, 2009 : 73-74).

\section{Les analyses proposées pour le génitif en wolof}

\subsection{Le génitif comme étant introduit par une préposition}

Dard (1826: 11-28) analyse le génitif wolof comme étant formé du nom tête suivi d'une préposition $u$, elle-même suivie du nom complément. L'auteur explique que, dans cette construction, le déterminant indéfini tombe. Boilat (1858:24-30) propose une analyse similaire du génitif wolof, qu'il nomme " complément du nom ». Il considère également que la détermination d'un nom par un numéral se forme de manière analogue au génitif.

19 (4a) fetal u naar ya

fusil PREP maure DF

'le fusil des maures'

20 (4b) i fetal u naar ya

i fusil PREP maure DF

'les fusils des maures'

\subsection{Le génitif comme étant introduit par un « article conjonctif »}

21 Senghor (1947) considère le $u$ comme un « article conjonctif ». Sa thèse est que cet article conjonctif n'est en fait rien de plus que le déterminant indéfini. Cette hypothèse est, selon lui, confirmée par certaines formes archaïques transmises par ses informateurs telles que :

22 (5a) as ndaw as tubaab

ART femme ART européen

'une jeune femme européenne' 
(5b) matt um Sangalkam bois ART Sangalkam 'bois de Sangalkam'

4 Dans ces exemples, l'article conjonctif est formellement identique au déterminant indéfini. Selon l'auteur, dans un état antérieur de la langue, le déterminant indéfini, aujourd'hui uniquement présent à gauche du nom, était répété à sa droite. Enfin, l'auteur note que l'article voit sa voyelle subir une élision lorsque le nom tête finit par une voyelle. Par ailleurs, il pose l'hypothèse que la forme $i$ de l'article conjonctif pluriel dérive du déterminant indéfini pluriel ay dont la voyelle est tombée, le y se vocalisant en $i$.

\subsection{Le génitif comme syntagme complétif}

Sauvageot (1965: 188-193) décrit le génitif, qu'il nomme « syntagme complétif », comme étant formé du nom tête auquel est attaché un «morphème fonctionnel », suivi du nom complément. Il note également qu'il ne peut y avoir aucun élément entre les deux noms hormis le morphème fonctionnel. Concernant ce qu'il nomme le «morphème fonctionnel », l'auteur remarque qu'il peut se réaliser sous deux formes, $u$ lorsque le nom tête est au singulier et $i$ lorsqu'il est au pluriel. Cependant, ce morphème peut être omis au singulier lorsque le nom tête est borom ('maître', 'propriétaire'), lorsque le nom tête finit par une voyelle, ou lorsque le nom complément est un nom propre. Enfin, l'auteur note qu'il existe une variante facultative de la construction génitive où l'on note la présence du marqueur de classe se rapportant au nom tête. Si le marqueur de classe est présent, le morphème fonctionnel peut être réalisé $u, i$ ou $a$, sans variation de sens.

\section{(6a) gétt-u xar}

troupeau-u mouton

'troupeau de moutons'

\section{(6b) gétt-u-g xar}

troupeau-u-CL mouton

'troupeau de moutons'

D'un point de vue syntaxique, l'auteur note qu'il est possible de combiner plusieurs « syntagmes complétifs » entre eux ; le degré de saturation semblant être atteint à partir de quatre syntagmes.

(7) gétt-i nag-i ñett-i doom-i Samba

troupeau-i vache-i trois-i enfant-i Samba

'les troupeaux de vaches des trois fils de Samba'

\subsection{Le génitif comme étant introduit par un « déterminant génitival »}

30 Diagne (1971: 98-100) parle de «déterminant génitival » introduit par les morphèmes $(w) u$ au singulier et $i$ au pluriel. L'auteur note que cette forme est compatible avec tous les déterminants.
(8a) doom-u jigéen $j i$
enfant-u femme la
'le fils de la dame' 
(8b) doom-u jigéen jan?

enfant-u femme quelle

'le fils de quelle femme ?'

(8c) doom-u benn jigéen

enfant-u une femme

'le fils d'une femme'

(8d) doom-u beneen jigéen

enfant-u une autre femme

'le fils d'une autre femme'

\title{
2.5 Le génitif comme détermination relationnelle par adjonction de nom
}

\begin{abstract}
Diouf (2009: 167-168) analyse la construction génitive comme une détermination relationnelle par adjonction de nom. Selon l'auteur, elle consiste à déterminer un nom ou un «nominal » par un autre nom. Si l'unité déterminée est au singulier et finit par une consonne, on utilise le suffixe connectif neutre - $u$. Cependant, avec l'individualisateur ${ }^{5}$ (à l'exception de menn), ou lorsque l'unité déterminée termine par une voyelle, il n'y a pas de connectif. Qu'il y ait ou non présence du suffixe connectif, on note la présence facultative de la marque de classe nominale de l'unité déterminée. Si l'unité déterminée appartient à la classe $j-$, on utilise la marque $-y$ à la place.
\end{abstract}

\subsection{Le génitif comme forme construite du nom}

Kihm (2000) soutient que la construction génitive du wolof est comparable à la forme construite du nom de plusieurs langues afro-asiatiques. Principalement parce que la construction génitive du wolof partage deux caractéristiques avec cette forme : elle est tête-initiale ; la valeur de définitude du complément génitif détermine celle du nom tête, qui est lui-même non marqué pour cette valeur. L'auteur explique que la construction génitive se forme en ajoutant un "suffixe complexe ", consistant en la voyelle u pouvant être suivie par le marqueur de classe, au nom tête. Le nom tête précède le syntagme génitif, ce qui est un premier point commun avec l'état construit du nom des langues afro-asiatiques. La variante avec le marqueur de classe n'est qu'une variante « littéraire » de la forme sans ce marqueur ; cette dernière consistant en une forme réduite de la première. Au pluriel, l'auteur considère que la forme $-i$ du suffixe correspond à la vocalisation du marqueur de classe pluriel $-y$ due à la chute de la voyelle $u$.

37 (9a) kër-u(-g) buur maison-u(-CL) roi

'une maison de roi'

(9b) kër-u(-g) buur bi

maison-u(-CL) roi le

'la maison du roi' (9c) kër-u-y buur bi

maison-u-CL.PL roi le

'les maisons du roi'

L'auteur explique que dans l'exemple (9a), ni le nom tête, ni le syntagme génitif ne sont définis, ce qui implique que le syntagme dans son ensemble est indéfini. Or, dans les 
exemples ( $9 b-c)$, seul le syntagme génitif est défini (le déterminant défini s'accorde en classe avec buur, non avec kër) ; là encore la définitude porte sur l'ensemble du syntagme. Ce point rapproche également la construction génitive du wolof de l'état construit du nom des langues afro-asiatiques. Le troisième point commun entre les deux constructions est le fait que le nom tête ne peut pas être directement modifié par quoi que ce soit. Par exemple, les verbes d'état (équivalents des adjectifs) doivent être placés à la droite du syntagme génitif. Par ailleurs, il est possible d'ajouter un modifieur du même type au syntagme génitif :

$40 \quad$ (10) kër-u(-g) buur bu ndaw gu mag maison-u(-CL) roi JONCT être jeune JONCT être grand

'les troupeaux de vaches des trois fils de Samba'

41 Comme en arabe ou en hébreu, il est possible d'enchaîner des génitifs en wolof. Dans ce cas, chaque nom constituera le syntagme génitif du nom qui précède, et seul le dernier nom peut être défini.

42 (11) kër-u(-g) buur-u(-b) réew mi gu mag maison-u(-CL) roi-u(-CL) pays le JONCT être grand 'la grande maison du roi du pays'

43 Selon l'auteur, le wolof respecte pleinement cinq des sept propriétés attribuées à l'état construit du nom. Une, la formation des composés n'est que partiellement respectée. Une autre, le statut du morphème lié et la chute de l'accent sur le premier terme (qui entraîne plusieurs processus phonologiques), ne semble pas être respectée du tout. Mais pour diverses raisons développées par l'auteur, cet état de choses n'est pas réellement problématique. L'auteur analyse le suffixe génitif comme un pronom de troisième personne renvoyant au nom tête. C'est ce pronom qui participe à la construction avec le syntagme génitif. Cette analyse permet de rapprocher la construction génitive du wolof avec l'état construit du nom indirect, qui est attesté dans plusieurs langues afroasiatiques.

44 L'une des conclusions de l'auteur, à l'issue de son étude, est que le u présent dans le suffixe génitif est une voyelle épenthétique servant de support au marqueur de classe nominale. Ce qui implique que ce marqueur de classe constitue le pronom évoqué plus haut. Cela est en accord avec un grand nombre de langues Niger-Congo, dans lesquelles les marqueurs de classe peuvent faire office de pronoms. Enfin, selon l'auteur, il existe trois configurations possibles concernant la définitude au sein d'une construction génitive en wolof: les deux termes sont définis (12a); les deux termes sont indéfinis (12b) ; l'un des termes est défini et l'autre est indéfini (12c-d).

45 (12a) këru(-g) buur bi maison(-CL) roi le 'la maison du roi'

$46 \quad$ (12b) kër-u(-g) (ag) buur maison(-CL) (une) roi 'une maison de roi'

47 (12c) ag kër-u(-g) buur bi une maison(-CL) roi le 'une maison du roi' 
maison(-CL) roi le

'la maison d'un roi'

\section{Analyse typologique du génitif wolof}

\subsection{Critique des analyses proposées et problèmes récurrents}

Les analyses proposées pour décrire le génitif en wolof posent plusieurs problèmes ${ }^{6}$ :

a. L'analyse s'appuie sur des exemples pouvant être sujets à caution ${ }^{7}$;

b. L'analyse ne tient pas compte de certaines réalités morphophonologiques de la langue ;

c. L'analyse ne rend pas compte de toutes les données ;

d. L'analyse n'a pas ou peu de portée typologique.

Le problème b), c'est-à-dire la non prise en compte de certaines réalités morphophonologiques de la langue n'est pas imputable à tous les travaux. On retrouve ce problème dans l'analyse du morphème $u$ par Dard (1826), Boilat (1858) et Senghor (1947). En effet, ces auteurs l'analysent comme un mot isolé, alors qu'il s'agit d'une unité phonologiquement faible. Par ailleurs, l'analyse des deux premiers auteurs est clairement un calque de l'analyse de la construction génitive en français : Nom tête + Préposition + Syntagme génitif. Diouf (2009) et Kihm (2000) l'analysent comme un suffixe, mais sans expliciter les raisons de ce choix. En nous appuyant sur les critères de Zwicky \& Pullum (1983), nous pouvons en effet dire que $u$ et $i$ sont des affixes en wolof : ils manifestent un haut degré de sélection par rapport à leur hôte (il ne peut s'agir que d'un nom); on note des lacunes arbitraires (le $u$ peut être omis après le mot borom, 'propriétaire' ${ }^{18}$ ) ; on note des idiosyncrasies morphophonologiques (si l'hôte finit par une voyelle, $u$ est omis); des règles syntaxiques peuvent affecter l'ensemble formé par le nom tête et le morphème $u$; ce morphème ne peut pas s'attacher à une séquence contenant des clitiques.

51 Le problème d), c'est-à-dire l'absence de portée typologique de l'analyse, concerne en premier lieu les appellations. En effet, celles-ci sont souvent idiosyncrasiques : « article conjonctif » (Senghor, 1947) ou « déterminant génitival » (Diagne, 1971). Ces étiquettes, créées de toutes pièces pour les besoins de l'analyse, résisteraient mal à une application à d'autres langues. Mais derrière ces étiquettes se trouve en réalité une analyse qui ne tient pas ou peu compte de la construction génitive dans d'autres langues. On pourrait avoir le sentiment que la construction génitive en wolof est typologiquement marquée, or, comme nous allons le montrer, il n'en est rien.

\subsection{La construction génitive wolof}

En nous basant sur les travaux précédemment évoqués, ainsi que sur les données transmises par nos informateurs, nous allons tenter de donner une description du génitif wolof prenant en compte toutes les données de la langue et ayant une portée typologique.

Comme nous l'avons vu plus haut, le génitif est formé à l'aide d'un morphème génitif suffixé au nom tête, immédiatement suivi par le syntagme génitif. La marque de génitif est $-u$ ou, plus rarement, $-a$ au singulier et $-i$ au pluriel. De plus, au singulier, le marqueur de classe du nom complété peut être ajouté à la marque de génitif. Cette construction est identique à la construction du déterminant indéfini, on peut donc avancer la même 
hypothèse pour l'opposition singulier/pluriel, à savoir que la chute du marqueur déictique $-u$ entraine une vocalisation du marqueur de classe $-y$. On observe des cas où la marque du génitif peut être omise au singulier : si le nom complété finit par une voyelle, si le nom tête est borom ('propriétaire'), ou si le syntagme génitif est constitué d'un nom propre. Par ailleurs, on constate une contrainte d'adjacence entre le nom tête et le syntagme génitif: il est impossible d'introduire le moindre élément entre ces deux constituants. Cette description nous permet d'ancrer la construction génitive du wolof dans l'un des types définis plus haut. Il s'agit d'une construction où le nom tête porte une marque du génitif qui est elle-même un affixe génitif invariable.

Cependant, certaines caractéristiques de la construction nous obligent à nuancer ce rapprochement. En effet, la possibilité d'indiquer la marque de classe sur le nom tête peut être la trace d'un état antérieur de la construction génitive. Senghor (1947) nous éclaire sur cet état en montrant l'analogie entre le suffixe génitif et le déterminant indéfini :

55 (13a) abxaj

un chien

'un chien'

$56 \quad(13 b) u b x a j$

un chien

'un chien'

57 (13c) xaj-ab buur

chien-GEN roi

'chien de roi'

58 (13d) xaj-ub buur

chien-GEN roi

'chien de roi'

59 Nous nous écarterons cependant de son analyse en refusant l'aspect synchronique qu'il lui confère. Nous considérons que la construction génitive actuelle en wolof est dans une phase transitoire entre un état où le génitif se marquait par le redoublement du déterminant indéfini ${ }^{9}$, et un état où il se marque par une marque du génitif suffixée au nom tête. La syntaxe du premier état est similaire à la syntaxe du génitif en grec ancien ${ }^{10}$ :

60 (14) ho híppos ho toû ánthrōpou

le.NOM cheval.NOM le.NOM le.GEN homme.GEN

'le cheval de l'homme'

61 Concernant l'état vers lequel le génitif wolof tend, nous pouvons dire qu'il s'agit plus précisément d'un état construit (construction rencontrée dans les langues sémitiques). En effet, comme l'a montré Kihm (2000), la construction génitive wolof respecte quatre des cinq principales propriétés de l'état construit : le nom précède le syntagme génitif; si le nom tête est modifié par une proposition relative, elle se trouve sur le bord droit de la construction; lorsque plusieurs syntagmes génitifs se succèdent, chacun d'entre eux, à l'exception du dernier, est une tête pour le syntagme génitif qui suit ; la relation entre le nom tête et le syntagme génitif n'est pas marquée par un élément tel qu'une adposition ou une marque casuelle.

62 Cependant, concernant la cinquième propriété, à savoir que la valeur de définitude $d u$ syntagme génitif s'étend sur l'ensemble de la construction, nous ne pouvons admettre une position aussi tranchée que celle de Kihm (2000). En effet, si l'on observe les exemples donnés par les différents auteurs, on constate un flottement concernant la définitude : 
l'exemple (4b) montre la possibilité d'avoir un syntagme génitif défini et un nom tête indéfini ; l'exemple (8c) montre la possibilité d'avoir un syntagme génitif ouvertement indéfini et un nom tête considéré comme défini. De plus, nos informateurs ne sont pas d'accord avec les traductions des exemples de Kihm (2000) représentant les configurations possibles concernant la définitude au sein d'une construction génitive en wolof. L'un de nos informateurs considère que les exemples (13a-b) peuvent être ou non marqués pour la définitude en fonction du contexte. Deux autres de nos informateurs considèrent les exemples (13b-c) comme agrammaticaux en raison de la présence du déterminant indéfini ag. À cause de tous ces contre-exemples, nous ne pouvons considérer que le wolof respecte cette propriété de l'état construit.

\subsection{Le possessif}

63 Le possessif se forme à l'aide de déterminants possessifs, sauf à la troisième personne du singulier où il est indiqué par un suffixe.

64 (15a) sama muus

POSS.1SG.SG chat

'mon chat'

65 (15b) muus-am

chat-POSS.3SG.SG

'son chat'

66 (15c) sa-y muus

POSS.2SG-PL chat

'tes chats'

67 (15d) seen-i muus

POSS.2PL-PL chat

'vos chats'

68 La construction possessive semble identique à la construction génitive. En effet, on peut rapprocher les constructions possessives du singulier avec les constructions génitives du singulier dans lesquelles la marque de génitif est omise ; et les constructions possessives du pluriel avec les constructions génitives du pluriel qui sont indiquées par la marque -i. De plus, les exemples $(15 \mathrm{c}-\mathrm{d})$ tendent à confirmer l'hypothèse concernant l'origine de la marque du génitif au pluriel. Cependant, la construction possessive ne saurait être assimilée au génitif pour une raison d'ordre syntaxique : dans la construction du génitif, le nom complété doit être immédiatement suivi par le nom complément, ce qui n'est pas le cas de la construction du possessif. En effet, le déterminant possessif peut apparaître soit avant le nom, soit après. Dans ce dernier cas, il ajoute une note affective (Diouf, 2009 : 161-162).

69 (16a) sama soppe

POSS.1SG.SG chéri

'mon chéri'

70 (16b) soppe sama

chéri POSS.1SG.SG

'mon tendre chéri'

71 Il semblerait plutôt que la possession soit marquée par un pronom possessif. En effet, on observe une très forte similitude entre les déterminants possessifs et les pronoms 
personnels objets : sama $\sim$ ma; sa $\sim$ la ; sunu $\sim$ nu; seen $\sim$ leen. Seul le marqueur possessif de troisième personne du singulier présente des particularités : il est suffixé alors que les autres sont des mots indépendants, et il ne présente aucun trait commun avec le pronom personnel objet équivalent. On peut cependant voir une analogie avec le pronom personnel sujet équivalent : $m u$. Il convient donc de considérer que, pour cette personne, la possession est marquée par un affixe possessif.

Enfin, l'une des particularités typologiques du wolof est que les énoncés comprenant un possessif et un génitif respectent la contrainte d'adjacence indiquée plus haut pour le génitif, mais séparent le déterminant possessif du nom qu'il détermine (Creissels, 2006 : 148).

73 (17a) sama fas-u nijaay POSS.1SG.SG cheval-GEN oncle 'le cheval de mon oncle'

74 (17b) sama fas-u nijaay wuñuul POSS.1SG.SG cheval-GEN oncle JONCT être noir 'le cheval noir de mon oncle'

75 Il convient de préciser qu'il est également possible d'insérer le possessif entre le nom tête et le syntagme génitif. Denis Creissels, cité par Kihm (2000 : 158), explique qu'il s'agit d'un «mauvais wolof» représentatif de locuteurs parlant le wolof comme une langue seconde. En revanche, l'un de nos informateurs (locuteur natif du wolof) considère qu'il s'agit uniquement de variantes.

76 (18a) sama kër-u xarit gi POSS.1SG.SG maison-GEN ami le 'la maison de mon ami'

77 (18b) kër-u sama xarit gi maison-GEN POSS.1SG.SG ami le 'la maison de mon ami'

$78 \quad$ (18c) sama kër-u baay-u xarit gi POSS.1SG.SG maison-GEN père-GEN ami le 'la maison du père de mon ami'

79 (18d) kër-u sama baay-u xarit gi maison-GEN POSS.1SG.SG père-GEN ami le 'la maison du père de mon ami'

$80 \quad$ (18e) kër-u baay-u sama xarit gi maison-GEN père-GEN POSS.1SG.SG ami le 'la maison du père de mon ami'

81 Si l'on admet la grammaticalité de ces exemples, nous pouvons poser l'hypothèse que la construction possessive du wolof est, comme la construction génitive, en cours d'évolution. Elle tend à être marquée par un pronom possessif (avec l'exception de l'affixe possessif à la troisième personne du singulier). Ce pronom possède plusieurs propriétés des déterminants : il peut se placer avant ou après le mot qu'il détermine, il se place à côté du nom du syntagme génitif. Néanmoins, comme nous l'avons montré plus haut, la construction possessive conserve des traces d'un état antérieur où elle était identique à la construction génitive. 


\section{Conclusion}

82

Malgré ce que les analyses proposées pourraient laisser penser, le génitif wolof ne présente pas d'originalités typologiques. Nous avons montré que cette construction est dans une phase transitoire entre un état où le génitif se marquait par le redoublement du déterminant indéfini (similaire à la syntaxe du génitif en grec ancien), et un état où il se marque par un suffixe sur le nom tête ; ce dernier étant similaire à «l'état construit du nom » attesté dans des langues sémitiques. Nous avons également avancé l'hypothèse selon laquelle en wolof la construction possessive (construction où le possesseur est une forme pronominale) est également en cours d'évolution. Elle est dans une phase transitoire entre un état où elle était identique à la construction génitive et un état où elle se marque par un pronom possessif.

\section{BIBLIOGRAPHIE}

Boilat, David (1858), Grammaire de la langue woloffe, Paris : Imprimerie Impériale.

Creissels, Denis (2001), « Catégorisation et grammaticalisation : la relation génitivale en mandingue ", in Robert NICOLAÏ (dir.), Leçons d'Afrique (hommage à Gabriel Manessy), Paris : Peeters, 433-454.

Creissels, Denis (2006), Syntaxe générale, une introduction typologique 1 : catégories et constructions, Paris : Lavoisier.

Creissels, Denis (2009), « Construct forms of nouns in African languages », in Peter Austin, Oliver Bond, Monik Charette, David Nathan \& Peter Sells (dir.), Proceedings of Conference on Language Documentation \& Linguistic Theory 2, Londres : SOAS, 73-82.

Dobrovie-Sorin, Carmen (2001), « Génitifs et déterminants », in Georges Kleiber, Brenda Laca, Liliane Tasmowski (dir.), Typologie des groupes nominaux, Rennes : Presses Universitaires de Rennes, 205-235.

Dard, Jean (1826), Grammaire wolofe, Paris : Imprimerie Royale.

Diagne, Pathé (1971), Grammaire de wolof moderne, Paris : Présence Africaine.

Diouf, Jean-Léopold (2009), Grammaire du wolof contemporain : Édition revue et complétée, Paris : L'Harmattan.

Dryer, Matthew (2007), « Noun phrase structure », in Timothy Shopen (dir.), Language typology and syntactic description 2: Complex Constructions, Cambridge : Cambridge University Press, 151-201.

Haspelmath, Martin (1999), « Explaining article-possessor complementarity: economic motivation in noun phrase syntax ", Language, vol. $75, \mathrm{n}^{\circ} 2,227-243$.

Corela, HS-16 | 2015 
Kihm, Alain (2000), « Wolof genitive constructions and the Construct State », in Jacqueline Lecarme, Jean Lowenstam, Ur Shlonsky (dir.), Research in Afroasiatic Grammar, Amsterdam/ Philadelphie : John Benjamins, 151-181.

Langacker, Ronald (1995), «Possession and possessive construction », in John Taylor, Robert MacLaury (dir.), Language and the cognitive construal of the world, Berlin : Mouton, 51-79.

Mahieu, Marc-Antoine (2013), «The Genitive Case and the Possessive Construction in Finnish », in Anne Carlier, Jean-Christophe Verstraete (dir.), The Genitive, Amsterdam/Philadelphie : John Benjamins.

Sauvageot, Serge (1983), Description synchronique d'un dialecte wolof: Le parler du Dyolof, Dakar : IFAN.

Senghor, Léopold Sédar (1947), « L'article conjonctif en wolof », Journal de la Société des Africanistes , vol. 17, $\mathrm{n}^{\circ} 1,19-22$.

Vernhes, Jean-Victor (2003), "Epualov : Initiation au grec ancien, Paris : Ophrys.

Zwicky, Arnold \& Pullum, Geoffrey (1983), « Cliticization vs. Inflection: English n't », Language, 59, 502-513.

\section{NOTES}

1. Le wolof est une langue atlantique (famille Niger-Congo) parlée en Sénégambie où il tend à devenir véhiculaire. Il présente une morphologie agglutinante ainsi qu'une syntaxe SVO. Comme de nombreuses langues Niger-Congo, il s'agit d'une langue à classes nominales.

2. Cette notion est à rapprocher de la notion, plus formalisée, de "point de référence » développée par Langacker (1995) dans le cadre de la grammaire cognitive.

3. Dans cet article, nous utiliserons les abréviations suivantes dans les gloses des exemples : \# = personne $; \mathrm{ADJ}=$ adjectif $; \mathrm{ART}=$ article $; \mathrm{CL}=$ marque de classe nominale $; \mathrm{DF}=$ défini $; \mathrm{GEN}=$ génitif $; \mathrm{JONCT}=$ joncteur $; \mathrm{NOM}=$ nominatif $; \mathrm{PL}=$ pluriel $; \mathrm{POSS}=$ possessif $; \mathrm{PREP}=$ préposition.

4. Si l'on se place dans le cadre de la grammaire générative transformationnelle, on peut dire que déterminant défini et construction génitive/possessive sont incompatibles car ils occupent la même position syntaxique, en l'occurrence [Spéc, N] (Dobrovie-Sorin, 2001).

5. L'individualisateur est le déterminant présentant «le nom comme se référant à une entité unique » (Creissels, $2006:$ 111).

6. Toutes les analyses ne posent pas forcément les quatre problèmes.

7. Nous ne traiterons pas ici des problèmes a) et c).

8. Cf. Sauvageot (1965: 189).

9. Dans un état antérieur de la langue, il existait peut-être d'autres marques du génitif, mais au regard des données dont nous disposons, il semble impossible de déterminer leur nature ou même leur existence.

10. Bien que, généralement, le complément du nom s'enclave entre le nom complété et son article (ho toû ánthrōpou híppos), cette construction est également attestée en grec ancien (Vernhes, 2003). 


\section{RÉSUMÉS}

Le génitif est une construction largement étudiée dans les langues, dont le wolof. Or, pour cette dernière langue, les analyses proposées n'ont pas de portée typologique. Au vu de ces analyses, on pourrait avoir le sentiment que la construction génitive en wolof est, d'un point de vue typologique, originale. L'auteur démontre, à partir d'une approche typologique, que la construction génitive en wolof est comparable à ce que l'on observe dans diverses langues appartenant à des familles linguistiques distinctes de celle du wolof. La construction génitive actuelle en wolof est dans une phase transitoire entre un état où le génitif se marquait par le redoublement du déterminant indéfini, et un état où il se marque par un suffixe sur le nom tête.

Genitive is a widely studied construction of languages, Wolof included. But, for Wolof, the proposed analyses have no typological reach. Reading these analyses, we could have the feeling that the genitive construction in Wolof is, from a typological point of view, unusual. The author demonstrates, from a typological approach, that the genitive construction in Wolof is similar to what we can observe in diverse languages belonging to linguistic families different from that of the Wolof. The current genitive construction in Wolof is in a transitional phase between a state where the genitive was marked by the redoubling of the indefinite determiner, and a state where it is marked by a suffix on the head noun.

\section{INDEX}

Mots-clés : génitif, possessif, syntaxe, typologie, wolof, syntagme nominal

Keywords : genitive, possessive, syntax, typology, noun phrase

\section{AUTEUR}

\section{MAXIMILIEN GUÉRIN}

Université Sorbonne Nouvelle - Paris 3

UMR 7528 - Mondes iranien et indien

UMR 8135 - Langage, Langues et Cultures d'Afrique Noire (LLACAN)

maximilien.guerin@univ-paris3.fr 